\title{
Investigation of pellet triggered MHD events in ASDEX Upgrade and JET
}

\author{
P.T. Lang, K. Lackner, M. Maraschek, B. Alper ${ }^{1}$, E. Belonohy ${ }^{2}$, \\ K. Gál' ${ }^{2}$, J. Hobirk, A. Kallenbach, S. Kálvin ${ }^{2}$,G. Kocsis² ${ }^{2}$ C.P. Perez von Thun, \\ W. Suttrop, T. Szepesi ${ }^{2}$, R. Wenninger, H. Zohm, \\ ASDEX Upgrade Team and JET-EFDA contributors ${ }^{\dagger}$
}

Max-Planck-Institut für Plasmaphysik, EURATOM Association, Boltzmannstr. 2, 85748 Garching, Germany

${ }^{1}$ EURATOM-UKAEA Fusion Association, Culham Science Centre, OX14 3DB, Abingdon, OXON, United Kingdom

${ }^{2}$ KFKI Research Institute for Particle and Nuclear Physics, EURATOM Association, P.O. Box 49, H-1525 Budapest-114, Hungary

${ }^{\dagger}$ See the Appendix of M. L. Watkins et al., Fusion Energy 2006 (Proc. 21st Int. Conf. Chengdu) IAEA (2006)

\begin{abstract}
To get deeper insight into the MHD activity triggered by pellets we extended our previous analyses of standard type-I ELMs to pellets injected into discharge phases of the following types: Ohmic, L-mode, type-III ELMy H-mode, ELM-free, radiative edge scenarios with type-I ELMs, the quiescent H (QH)mode regime. It turns out that pellet injection generally creates a strong, local perturbation of the MHD equilibrium in the ablation region and even beyond. Regarding the triggering of ELMs, this initial perturbation can damp out, indicating that the plasma is stable in the corresponding regime even for finite-size perturbations. This behaviour is observed not only in Ohmic and L-mode phases but also in the QH-mode where the edge harmonic oscillations (EHO) appear to keep the edge within or at the boundary of a stable regime. In case the plasma is prone to ELM growth, the large amplitude of the pellet perturbation can trigger the event even in situations where modes appear to be still linearly stable. The non-linear character of the ELM trigger process is highlighted also by the subsequent explosive growth of these events. For edge plasma conditions characterised by higher resistivity the growth time of spontaneously occurring ELMs increases when the plasma changes from the type-I into the typeIII regime. Pellet-triggered ELMs, however, maintain the fast rise times otherwise typical for the hot edge type-I regime. In the discussion section we attempt to relate these observations also to core mode activity like neoclassical tearing modes or snakes. Data were taken from ASDEX Upgrade and JET. Pellet triggering of mode activity can be shown to be a quite universal phenomenon, which, however, only for the case of ELMs can be unambiguously attributed to prompt direct excitation by the pellet.
\end{abstract}

PACS numbers: 52.35.Py, 52.55.Fa, 52.55.Tn 


\section{INTRODUCTION}

Edge localised mode (ELM) induced erosion of plasma facing materials has been recognised as a possible threat to the viability of ITER [1]. Several approaches to solve this problem are currently under investigation. One possible option is to establish ELM control via the pacing concept, by raising, in a controlled manner the ELM occurrence frequency, $f_{E L M}$, above its natural value, $f_{E L M}^{0}$, thereby reducing the energy per ELM $\Delta W_{E L M}$ according to the empirically observed relation $f_{E L M} \times \Delta W_{E L M}=$ const. [2]. ELM pacing by the injection of small pellets has been demonstrated successfully over a broad parameter range. Scenarios with controlled ELMs can satisfy even such diverse requirements as impurity expulsion (during intrinsically ELM-free phases) on one hand, and the reduction of impulsive divertor heat loads on the other hand [3]. To gain insight into the more detailed physics of ELM triggering (and ultimately also into its scaling), we have analyzed pellet injection into different types of discharges. We tested the hypothesis that an injected pellet excites always a helical perturbation, either by the local interruption of the helical equilibrium current in the edge zone, or by the localized change in plasma pressure. Depending on the stability properties of the pre-injection plasma this excitation should either result in damped oscillations (like the ringing of a chord) or a growing perturbation.

Results of such experiments during Ohmic $(\mathrm{OH})$ and L-mode plasmas as well as in ELM-free, type-III and type-I ELMing and QH-modes confirm this picture. Amongst the investigated scenarios we included also two in which an enforced enhancement of the edge resistivity resulted in an increased growth time of the spontaneously occurring ELMs. The first stayed in the original type-I regime but with a cooled radiative edge shell established by Argon puffing, the other entered ultimately the type-III regime, driven by edge cooling via enhanced gas puffing. In the $\mathrm{OH}$ and L-mode regime, pellet injection allowed for the study of the MHD perturbation directly driven by the pellet imposed impact on the plasma since the chosen configurations are not prone to release and continuance of an intrinsic MHD mode in the plasma. Data used in this study are from recent experiments performed on ASDEX Upgrade (AUG) dedicated to ELM triggering but also from previous investigations on AUG and JET using pellet injection for other purposes (mainly fuelling). The analysis of the latter discharges is partly hampered by the not fully optimized pellet and/or diagnostics settings but the results can nevertheless be used to show the main features of the pellet impact.

\section{EXPERIMENTAL SETUP}

Most of the analyzed experiments were performed during the last decade on AUG, a mid-sized divertor tokamak with torus radius $R_{0}=1.65 \mathrm{~m}$, minor plasma radius $a_{0}=0.5 \mathrm{~m}$, a typical plasma elongation $\frac{b}{a}>1.6$, and a plasma volume $V_{\text {Plasma }}=13 m^{3}$ [4]. During this period the divertor geometry and the wall material composition have undergone several revisions. The experimental configurations and plasma scenarios of the individual discharges considered are described either briefly in the corresponding sections here or in the documentation of the first analyses of these experiments. 
The injector employed is based on a centrifuge pellet accelerator equipped with a storage cryostat pellet reservoir [5]. It is capable of delivering up to 120 cubic pellets of nominal size $(1.4 \mathrm{~mm})^{3}$ to $(1.9 \mathrm{~mm})^{3}$ (nominal pellet masses $m_{P}$ corresponding to a particle inventory ranging from 1.6 to $4.110^{20} \mathrm{D}$-atoms) at velocities $v_{P}$ from $240 \mathrm{~m} / \mathrm{s}$ to $1200 \mathrm{~m} / \mathrm{s}$ and repetition rates $f_{P}$ up to $80 \mathrm{~Hz}$. During the period considered, the injection geometry has undergone several modifications, and the information on the precise version used for a specific experiment can to be found in the references provided.

Diodes detecting the $D_{\alpha}$ radiation from the plasma region including the pellet path yield a monitor signal for the pellet ablation. To provide knowledge of the pellet position at any time in spite of the possible bending of their trajectories by the ablation process a fast framing camera system has recently been installed [6]. The MHD mode activity in the plasma is monitored by Mirnov coils recording different magnetic field components with high temporal resolution.

A set of 30 coils mounted to the wall of the vacuum vessel measure the poloidal magnetic field component distributed around the entire poloidal circumference. Printed circuit coils measuring the radial magnetic field component are mounted on the magnetic low field side only about $0.1 \mathrm{~m}$ from the separatrix at different poloidal and toroidal positions. They are particularly suited to study high frequency MHD phenomena like the ELM onset and cycle. Together, covering different poloidal, toroidal and radial positions these diagnostics provide a good overview of the global structure of the MHD perturbation. In addition to specifically mentioned systems AUG is equipped with a broad range of general diagnostics [4].

For JET we also re-examined results of earlier experiments dedicated mainly to fuelling. Results from a recent dedicated ELM trigger study on previous JET experiments [7] complemented our data. They cover pellet injection into type-I and ELM-free H-modes of a large divertor tokamak $\left(R_{0}=2.96 \mathrm{~m}, a_{0}=1.25 \mathrm{~m}\right.$, $\left.V_{\text {Plasma }}=80 \mathrm{~m}^{3}\right)$. Again, the details of the experimental set up, pellet and plasma parameters can be found in the corresponding references. Furthermore, first data could be achieved very recently using the new JET pellet launching system [8] during commissioning tests.

\section{STRATEGY}

To test our hypothesis regarding the crucial role of the pellet-excited helical MHD perturbation for mode destabilisation, we analysed the impact of pellet injection on the mode activity for a broad range of scenarios. Our prime interest was in triggered edge localized modes but to identify more universal features we studied also again the already known impact of pellet injection on core modes. As reference for purely pellet-driven MHD perturbations we used discharges considered immune against spontaneous edge mode activity. Such conditions are expected in plasmas operated well below the $\mathrm{L} \rightarrow \mathrm{H}$ threshold at low heating power, with little internal energy available to drive instabilities. Gradually increasing heating power and plasma energy makes plasmas more prone to spontaneous but also triggered mode growth. At the same time the evolution of the MHD activity following pellet injection is expected to become dominated less by the onset conditions and more by the growth dynamics of the triggered mode. 
The systematic of the observed ELM behaviour is dominated by the power flux through the separatrix $P_{S e p .}$. [9] and hence roughly preset by the applied heating power $P_{H}$. The studies reported in this paper follow essentially this systematic: For baseline plasma type operation we start with $\mathrm{OH}$ discharges as a vehicle to study the pure, directly pellet-induced MHD activity. In this $\mathrm{OH}$ regime we investigated the impact of changing the initial pellet perturbation by variation of $v_{P}$ and/or $m_{P}$. L-mode plasmas with moderate auxiliary heating, still regarded stable against ELM activity, were then employed to study the influence of plasma parameters on the pellet induced MHD perturbation.

Crossing the $\mathrm{L} \rightarrow \mathrm{H}$ transition threshold the plasma enters a regime characterized by a strong transport barrier at the edge resulting in a pedestal with a steep gradient zone. These steep gradients in the plasma pressure and current density profiles are considered responsible for the cyclic appearance of the peelingballooning instability underlying the periodic ELM events $[10,11,12]$. Following the $P_{S e p}$. oriented ELM classification [9] for baseline scenarios we enter first the type-III regime $\left(\frac{d f_{E L M}}{d P_{S e p}}<0\right)$ and reach, with increasing $P_{S e p}$. finally by $f_{E L M} \rightarrow 0$ the ELM-free regime. Usually, ELM-free phases are not stationary and usable for steady-state operation as e.g. the continuous profile evolution into an unstable regime and/or impurity accumulation terminate such phases. Proceeding beyond the ELM-free regime to still higher $P_{S e p}$. results in type-I ELM dominated discharges $\left(\frac{d f_{E L M}}{d P_{S e p} .}>0\right)$.

Besides these regimes observed generally and reproducibly in baseline scenarios there exist several others plasma regimes - often accessible by applying very special operational conditions - usually characterized by narrow operational area or limited reproducibility. As an example from this category we report here on the QH mode.

In the discussion section we briefly relate these observations also to other cases of pellet-triggered macroscopic perturbations, like NTMs and snakes.

\section{RESULTS}

During the investigations, we mainly focussed on the following three issues:

i) what is the magnitude of the local perturbation directly imposed by the pellet,

ii) what are the characteristics of the MHD perturbation directly driven by it,

iii) and what is finally the general response of the plasma MHD evolution.

To allow the isolated investigation of the perturbation directly driven by the pellet, injection into the $\mathrm{OH}$ and L-mode plasmas is particularly well suited. These plasmas not prone to release and continuance of an intrinsic MHD mode do allow to study the effect stemming from the pellet induced impact. All local changes in plasma parameters are than ultimately driven by the ablated cold pellet particles and should therefore respond in magnitude to the $\dot{m}_{P}(t)$ evolution. As a monitor for the pellet ablation we adopt the diode recorded ablation radiation $D_{\alpha}$ signal. This radiation intensity is known to represent fairly well the time-varying pellet particle ablation rate $\frac{d m_{P}}{d t} \equiv \dot{m}_{P}$ [13]; this approximation was also well confirmed by our earlier pellet ELM pacing and fuelling studies [14]. As indicator for the magnitude of

the MHD perturbation we took measurements of $\frac{d B_{\Theta}}{d t} \equiv \dot{B}_{\Theta}$ and $\frac{d B_{r}}{d t} \equiv \dot{B}_{r}$ by the Mirnov coils. Our 
recent investigations on spontaneous and triggered ELMs showed that any of these coils displays the ELM onset fairly well. The first indication of the sharp increase caused by a type-I ELM in the MHD signal is randomly distributed all around the torus and observed within $20 \mu s$ in every coil signal. This was interpreted as result of fast shear Alfvén wave communication along magnetic field lines all around the torus of an ELM with an undetermined onset position, also in case of triggered ELMs [15]. To monitor the MHD perturbation we hence either picked a suitable single signal trace or took an average. The amplitude of the Mirnov signal is dependent on distance, frequency and amplitude of the ongoing B perturbation. Due to this coupling, reconstructing location, extension and magnitude of the underlying B perturbation structure would require extensive modelling.

For this study we simplify the picture assuming in the ELM case the Mirnov signal is stemming from the mode activity in the edge region closest to the coil. In the pellet case, we assign the directly driven MHD signal to the flux surface just visited by the pellet. To characterise the plasma response, and in particular to answer the question whether an ELM was actually triggered, we employed well known indicators like the energy pulse to the strike points visualised by distinct spikes in the divertor $D_{\alpha}$ radiation or the drop in edge particle and pressure content.

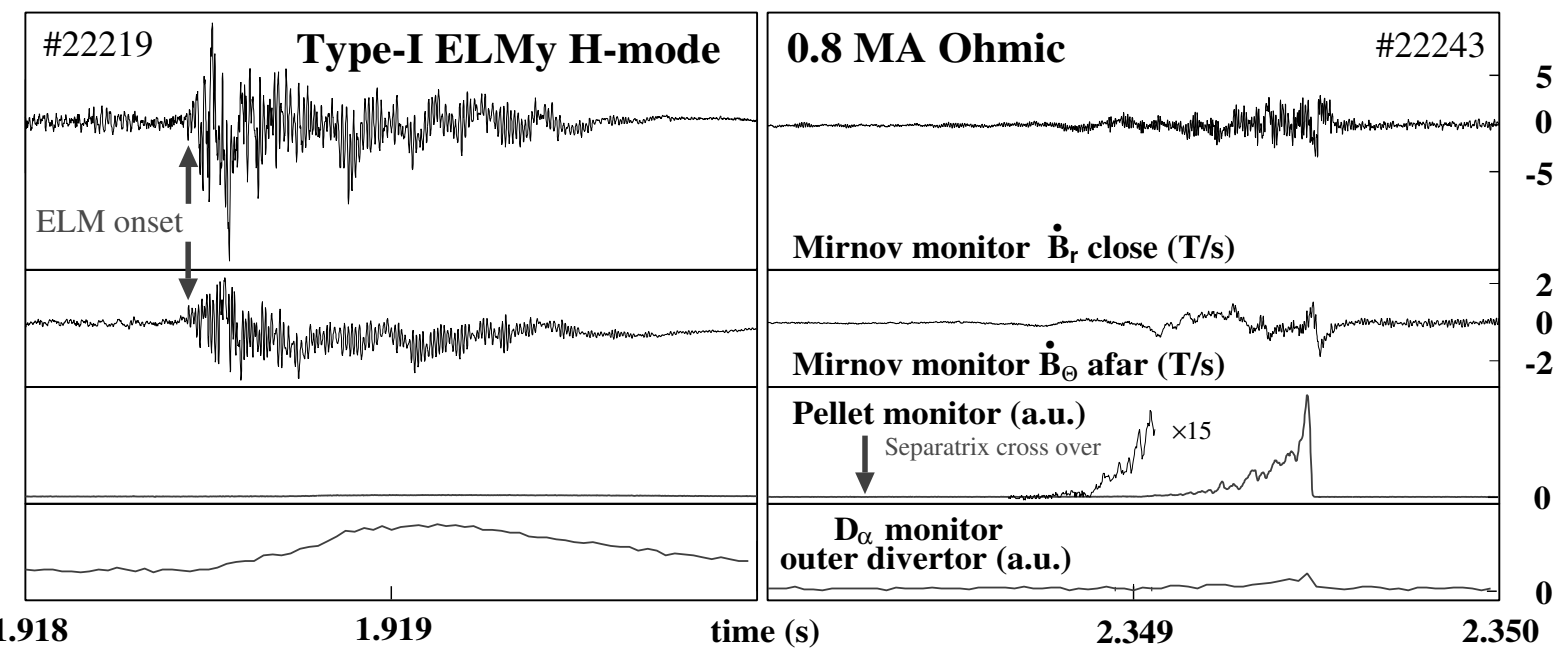

Figure 1: MHD perturbations detected by Mirnov monitors during a spontaneous type-I ELM and pellet injection into $\mathrm{OH}$ plasma (identical scales and diagnostics settings). The shown pellet driven MHD perturbation ( $\mathrm{OH}$ reference case) represents the weakest impact observed over the range of technically available pellet parameters. Nevertheless, it exceeds the magnitude of the MHD activity observed at the very onset of the ELM as indicated by the arrows.

4.1. Purely pellet driven magnetic perturbations in edge stable regimes below the $\mathrm{L} \rightarrow \mathrm{H}$ threshold As a first benchmark we tested in AUG whether the directly pellet driven perturbation of the magnetic equilibrium is potentially strong enough to explain the ELM trigger. For this we compared the perturbation magnitude of the magnetic field at the first detectable onset of a spontaneous type-I ELM (a triggered one looks virtually identical) to the directly pellet driven perturbation in a regime not prone 
to ELMs - an OH plasma with low plasma energy. As can be seen clearly in figure 1 the directly pellet driven magnetic perturbation is stronger than that one observed at the time the ELM event can be clearly recognized (as indicated by the arrows). It is worth noting that the $\mathrm{OH}$ pellet case shown is one of the faintest excitations observed in the study (small $240 \mathrm{~m} / \mathrm{s}$ pellet). The type-I reference discharge chosen was close in time to it, so as to minimize diagnostic trim differences. Displayed are (from top to bottom) perturbation monitor signals from $\dot{B}_{r}$ and $\dot{B}_{\Theta}$ Mirnov coils, $D_{\alpha}$ pellet and ELM monitor. The latter like other ELM monitors as well - shows the typical type-I ELM behaviour in the H-mode reference case while clearly no such signature is present in the $\mathrm{OH}$ case. The marginal response in the $\mathrm{OH}$ case is due to transiently enhanced convective losses after the pellet particle deposition has modified the profiles. When the pellet enters the plasma mode activity sets in (the separatrix crossing was determined from the fast framing camera trajectory reconstruction, details in next section). The magnetic perturbation decays quickly (within about 50 $\mathrm{s}$ - which is about the same time needed to trigger an ELM after the corresponding perturbation has been imposed [15]) once the driving pellet ablation vanishes after pellet burn out. Hence, it is quite obvious the magnetic perturbation is driven purely by the pellet and no growing mode activity was triggered.

There is a clear correlation between pellet ablation and this directly driven magnetic response. Both displayed Mirnov monitor signals show the same basic trend: an increase in magnitude while the pellet penetrates deeper into the plasma and $\dot{m}_{P}$ increases also. Mounted closer to the separatrix the $\dot{B}_{r}$ Mirnov coil shows a stronger contribution of higher m,n mode components while the more distant $\dot{B}_{\Theta}$ Mirnov coil is dominated by the slower-decaying low m,n-modes. From these benchmark experiments it is expected that the directly pellet driven magnetic perturbation could trigger an ELM in a suitably unstable plasma. The Mirnov monitor signal can be used to investigate the correlation between the directly pellet driven perturbation $\dot{m}_{P}$ and the ultimate plasma response (rapid declining perturbation after pellet burn out or triggering of an MHD mode). To find out if there is a correlation between the pellet imposed perturbation $\dot{m}_{P}$ and the directly driven perturbation obviously a regime without significant intrinsic or overlaying triggered MHD activity is required. Thus, in a first exploration we analysed the direct pellet driven impact in a few $\mathrm{OH}$ and low power L-mode phases. A dedicated scan comprising 4 discharges was performed launching pellets of different mass and/or velocity into the standard $\mathrm{OH}$ discharge (0.8 MA, Bt $=-2.5 \mathrm{~T}$ ) run routinely at AUG plasma operation start up. A few pellets (typically $5-10$ with identical initial $m_{P}$ and $v_{P}$ ) were injected during the flattop phase of each discharge at very low repetition rates $\left(f_{P}\right.$ approx. $\left.5 \mathrm{~Hz}\right)$ causing only transient perturbations. Hence, all pellets in a single discharge can be regarded as repeatedly performed (virtually) identical experiments.

In different discharges, different pellet velocities $(240,600$ or $880 \mathrm{~m} / \mathrm{s})$, and different pellet sizes - further referred to as small, medium or large - were used (nominal launch $m_{P}$ due to a particle inventory of 1.7, 2.8 or $4.3 \times 10^{20} \mathrm{D}$, - however the mass fraction actually arriving at the plasma degrades with increasing speed). The $\mathrm{OH}$ reference case shown in figure 1 is taken from this scan.

A simple but robust method was applied to monitor the correlation between pellet ablation parameters and the driven MHD perturbations. Initially single characteristic coil signals were compared, while later 


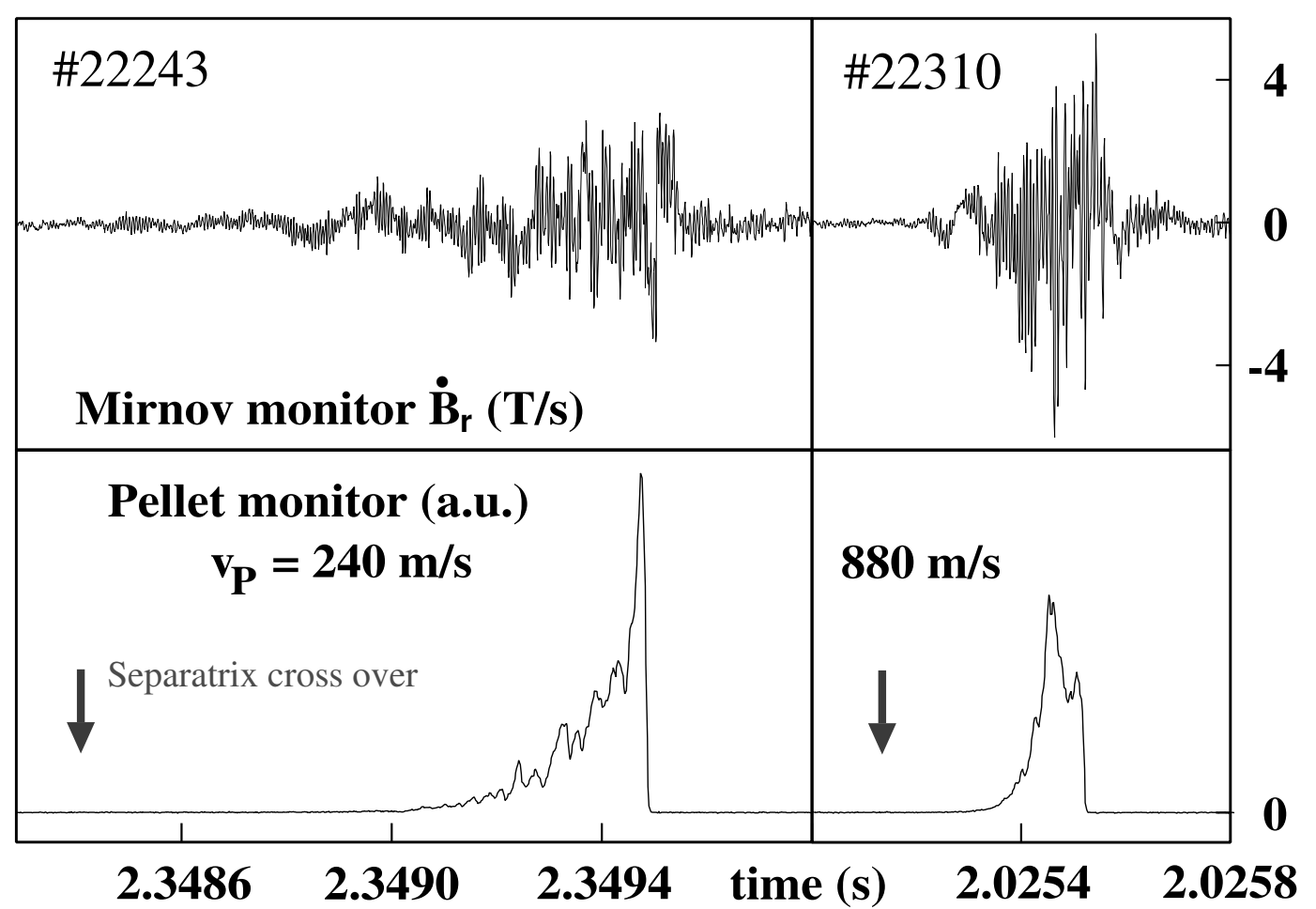

Figure 2: Signal traces representing pellet ablation and resulting MHD perturbation as applied in the analysis for most extreme cases available - a small $240 \mathrm{~m} / \mathrm{s}$ and a large $880 \mathrm{~m} / \mathrm{s}$ pellet.

averaged signals were used. Signals from a dedicated $\dot{B}_{r}$ Mirnov coil (outboard horizontal mid plane) providing reliable data for all relevant pellets and an ablation monitor showing no indication of saturation were selected. Examples of these signal traces recorded for two pellets, a large fast and a small slow one, are shown in figure 2 .

In principle, the evolution of the magnetic field amplitude would be more feasible to study. However by integration of the Mirnov signal slow changes probably due to variations of the entire plasma equilibrium were introduced making the analysis more troublesome. Hence we refrained from calculating the evolution of $\mathrm{B}(\mathrm{t})$ and analysed directly the pure Mirnov data. The Mirnov signal consists mainly of frequency components around $150 \mathrm{kHz}$, around $20 \mathrm{KHz}$ and at still lower frequencies. The latter are attributed to plasma pressure profile changes and are not relevant for the present investigation. We therefore filtered them out by using the following simple box car method: For every pellet, the signal was segmented into short time slices (typical $20 \mu \mathrm{s}$ ) and the difference between minimum and maximum values in each slice was calculated. The resulting value $\dot{B}_{r p}(t)$ gives the variation in time of the amplitude of the high frequency signal for each pellet, and is in turn modulated by the medium frequency component thought to be caused by the spatial structure of the rotating perturbation and its varying distance to the coil. As the onset of this structure is of statistical nature, we can average over all $\dot{B}_{r p}(t)$ time slices for all pellets in an entire discharge and arrive thus at the signal $\left\langle\dot{B}_{r p}\right\rangle(t)$. This single curve for each discharge provides then a good measure for the temporal evolution of the magnetic perturbation magnitude for the 
entire pellet ensemble $\left(<\dot{B}_{r p}>(t)\right.$ corresponds to about 0.7 times the envelope of all the single $\dot{B}_{r p}(t)$ curves).

A critical point already for the averaging within one sample but even more for the correlation between different samples is the assignment of time and pellet position. This time was identified using a framingcamera-based pellet trajectory reconstruction. It turns out in these $\mathrm{OH}$ discharges pellets follows straight their designated trajectory without significant change in velocity. However, detectable ablation radiation can be observed in the diode monitor and the camera systems (fast framing and long exposure video observation) only once the pellet has penetrated along the pellet trajectory about $5-10 \mathrm{~cm}$ beyond the separatrix. This is an unexpected and yet unexplained result. A preliminary investigation however indicates that in purely NBI heated H-mode discharges strong ablation radiation sets in also only significantly beyond the separatrix while additional electron cyclotron heating causes a corresponding onset already a few mm outside the separatrix. A more detailed study covering a broader range of pellet and plasma parameters by taking data from the HFS-PAD database [16] is now launched and under way. Nevertheless the $D_{\alpha}$ ablation monitor can still be used as relative measure whose reliability for the subsequent major part of the pellet trajectory was confirmed by the still observed correlation $\int D_{\alpha} \sim \int \dot{m}_{P} \sim \widetilde{m}_{P} \sim \Delta N_{\text {plasma }}\left(\widetilde{m}_{P}\right.$ estimated pellet mass arriving in plasma taking into account tube transfer losses [17], $\Delta N_{\text {plasma }}$ plasma particle inventory enhancement by injection).

An attempt was now made to relate the pellet ablation to the driven MHD perturbation. For a given set of $v_{P}$ and $m_{P}, \dot{m}_{P}(t)$ and $\dot{B}_{r p}(t)$ (or $<\dot{B}_{r p}>(t)$ ) were found to be well related. Changing of the pellet parameters, however, modifies this relation: e.g. increasing $v_{P}$ results in larger values of $\left\langle\dot{B}_{r p}\right\rangle(t)$ for a given $\dot{m}_{P}$, as is shown in figure 3 .

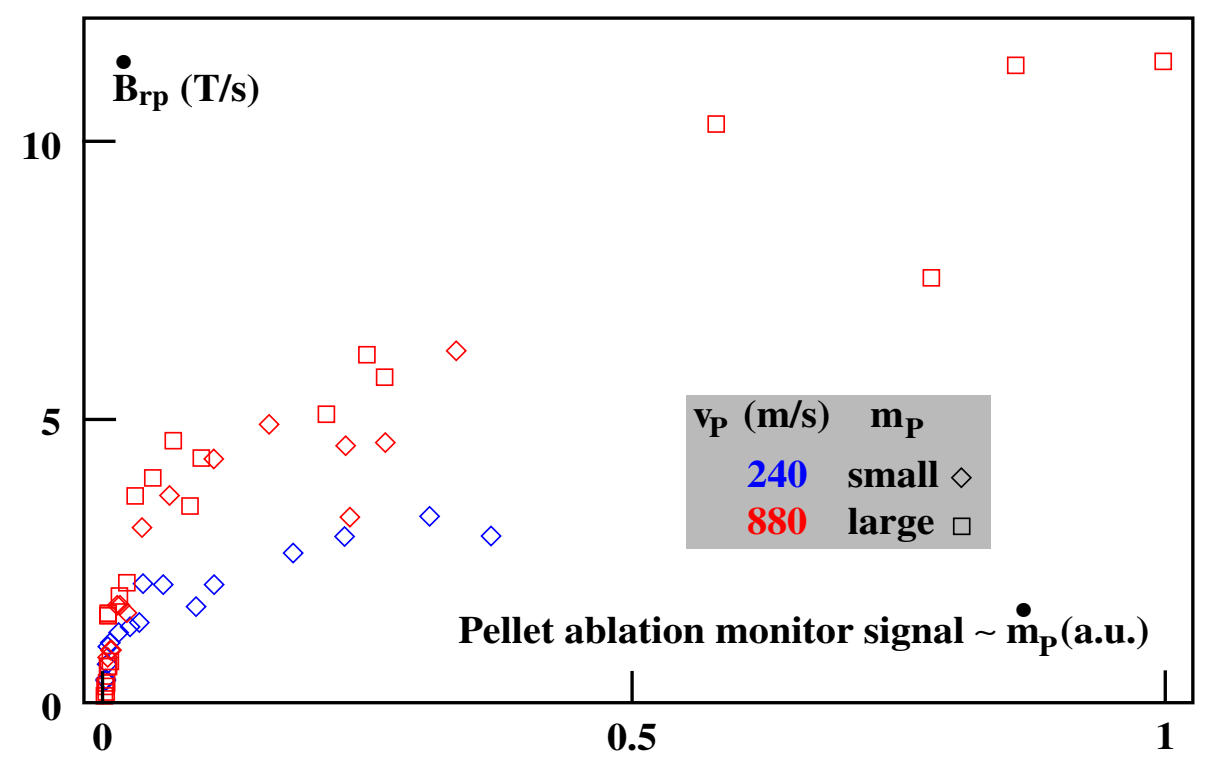

Figure 3: Pellet induced magnetic perturbation $<\dot{B}_{r p}>$ versus pellet ablation rate $\dot{m}_{P}(t)$ at different pellet speed $(240$ and $880 \mathrm{~m} / \mathrm{s})$. The pellet driven MHD perturbation is neither directly related to the pellet ablation rate (as can be concluded from the data shown) nor the deposition rate (see text). 
One could argue that what matters is the total amount of particles deposited in a reference volume rather than the ablation rate. However, replacing the temporal ablation rate $\dot{m}_{P}\left[s^{-1}\right]$ by the spatial deposition rate $\frac{d m_{P}}{d r}\left[m^{-1}\right] \equiv m_{P}^{\prime}$ (using the relation $m_{P}^{\prime}=\frac{\dot{m}_{P}}{v_{P}}$ ) even increases further this mismatch.

Failing to find a direct relation between $<\dot{B}_{r p}>(t)$ and the pellet parameters, we investigated the relation to the local plasma parameters. However, since injection was performed into virtually identical plasmas, data from the pellet parameter scan could only explore the relation with the pellet position in the plasma. The result is shown in figure 4 , plotting $\left\langle\dot{B}_{r p}\right\rangle$ evolutions observed for different $v_{P} / m_{P}$ samples, versus the pellet position when advancing along the injection path with the separatrix intersection defined as 0 . For all pellet data there is a clear trend for an increasing $\left\langle\dot{B}_{r p}\right\rangle$ with the pellet penetration deeper into the plasma. Also only the local plasma parameters appear relevant for the magnitude of the pellet triggered perturbation while changes in the ablation/deposition rates imposed by altered pellet parameters do not show a significant effect. Large/fast pellets create a stronger magnetic perturbation compared to small/slow ones only in that part of their ablation region located deep inside the plasma not reached by the small/slow ones. At a given plasma position and hence for the same plasma parameter, however, the same magnetic perturbation is generated by the smallest and largest pellet impacts tested.

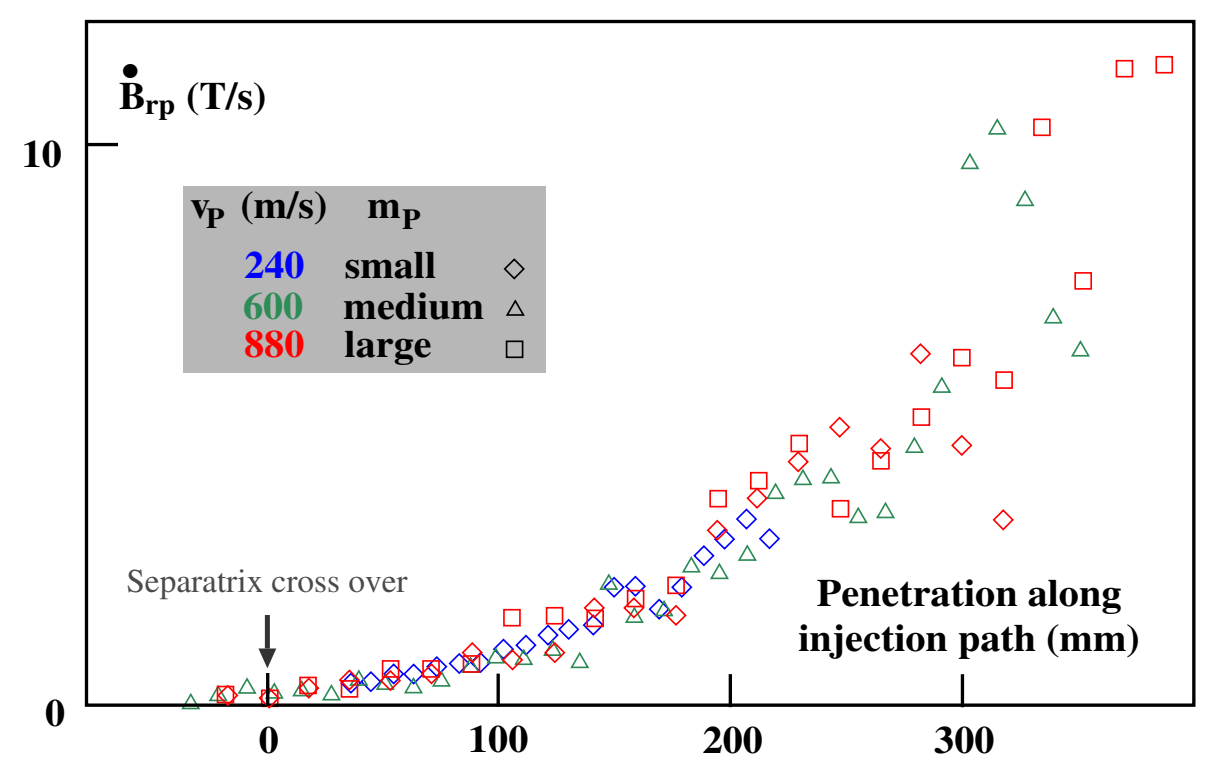

Figure 4: Pellet induced MHD perturbation $\left\langle\dot{B}_{r p}>\right.$ versus pellet position along the injection path, separatrix position defines 0 . At the same position in the plasma, the same magnitude for the pellet driven MHD response is observed for any pellet speed and/or mass applied (inserted legend, \#22243 small 240 m/s; \#22559 medium 600 m/s; \#22310 medium 880 m/s; \#22537 large 880 m/s).

Obviously, in the parameter regime investigated pellet particle ablation or deposition rates do not matter with respect to the magnitude of the resulting magnetic perturbation. As no direct relation of the driven perturbation strength to the underlying pellet born perturbation was found it became clear that the driving mechanism reaches already at every plasma position a saturated regime and the deposition of 
more pellet particles cannot provoke a stronger Mirnov activity. This implies that there should be still headroom for a reduction of the ablation/deposition rates by injecting smaller/faster pellets while keeping the full drive on the resulting perturbation.

For a reasonable interpretation of the pellet induced perturbation of course detailed information on the nature and structure of this MHD activity is required. Such information is available from the temporal behaviour of the Mirnov signal frequency power spectrum. An example given in figure 5 shows the frequency amplitude evolution from a Fourier frequency analysis (200 $\mu$ s rectangular sliding time window) of a horizontal mid plane LFS Mirnov coil signal. Pellet injection (example shown in right part of figure 2) causes a clearly noticeable event.

It consists of a low frequency (up to a few $10 \mathrm{kHz}$ ) component and toroidicity induced Alfvén eigenmode (TAE)-like sub structures in the region between 100 and $200 \mathrm{kHz}$. With toroidal mode numbers $(n \geq 5)$ consistent with the Alfvén gap structure, the pellet driven MHD activity looks like an enhancement of drift Alfvén turbulence driven TAE at the plasma edge in the vicinity of $\rho_{p o l}=0.8$. The usual L-mode Alfvén spectrum shows a clear, but always continuum damped TAE resonance $\left(f_{T A E}(\rho)=\frac{\left(\frac{v A(\rho)}{2 q(\rho) R}\right)}{2 \pi}\right.$ is strong monotonic, with $v_{A}(\rho)$ the Alfvén velocity at the according location). This resonance is typically located at $\rho_{\text {pol }}=0.8$ according to CASTOR calculations and as measured repeatedly in L-mode plasmas [18]. The calculated damping rates of these modes indicate the strong continuum damping.

This kind of modes was first reported in ASDEX Upgrade and explained by a two step excitation mechanism $[18,19]$. Due to the coupling at short perpendicular wavelength shear Alfvén waves are excited in the plasma edge by electron drift turbulence. This short wavelength Alfvén activity is then transferred into global-scale modes via the $2 \mathrm{D}$ inverse cascade [20]. With these characteristics the intermediate broad band transient event around $150 \mathrm{kHz}$ resemble an ELM pattern [21]. The observed frequency drop of this intermediate-frequency activity is caused by the local density increase imposed by the deeply penetrating pellet in the vicinity of $\rho_{\text {pol }}=0.8$. The correlation of frequency drop with density increase is also consistent with the frequency-density scaling $f\left(\rho_{p o l}\right) \sim \frac{1}{\sqrt{n_{e}\left(\rho_{p o l}\right)}}$ reported for this modes. Present already weakly before the pellet it is enhanced in amplitude by more than a factor of 100 by the pellet and than declines rapidly to its initial strength.

To get information on localisation, extent and structure of the pellet enhanced MHD activity, correlation and a mode analysis were performed. A standard cross-spectrum calculation as used in [22] for pairs of magnetic probes made evident the strong correlation caused by the global structure of the mode as shown in figure 6. There, the cross-power spectrum of two Mirnov signals - the one used in the preceding analysis (figure 5) and a second one from another mid plane LFS Mirnov coil displaced by 3 sectors (toroidal distance $3 \times \frac{2 \pi}{16} \approx 68^{\circ}$ ) - is displayed for a time window covering the entire pellet lifetime. The strong correlation in both the low and intermediate frequency ranges during the phase of strong activity indicates a coherent mode. Again we derived toroidal mode numbers of the order 5-6 for the medium frequency components, consistent with TAE activity at the plasma edge. This belief is further supported by SXR data indicating for a location of the main MHD activity in the region around $\rho_{\text {pol }}=0.75$. A 


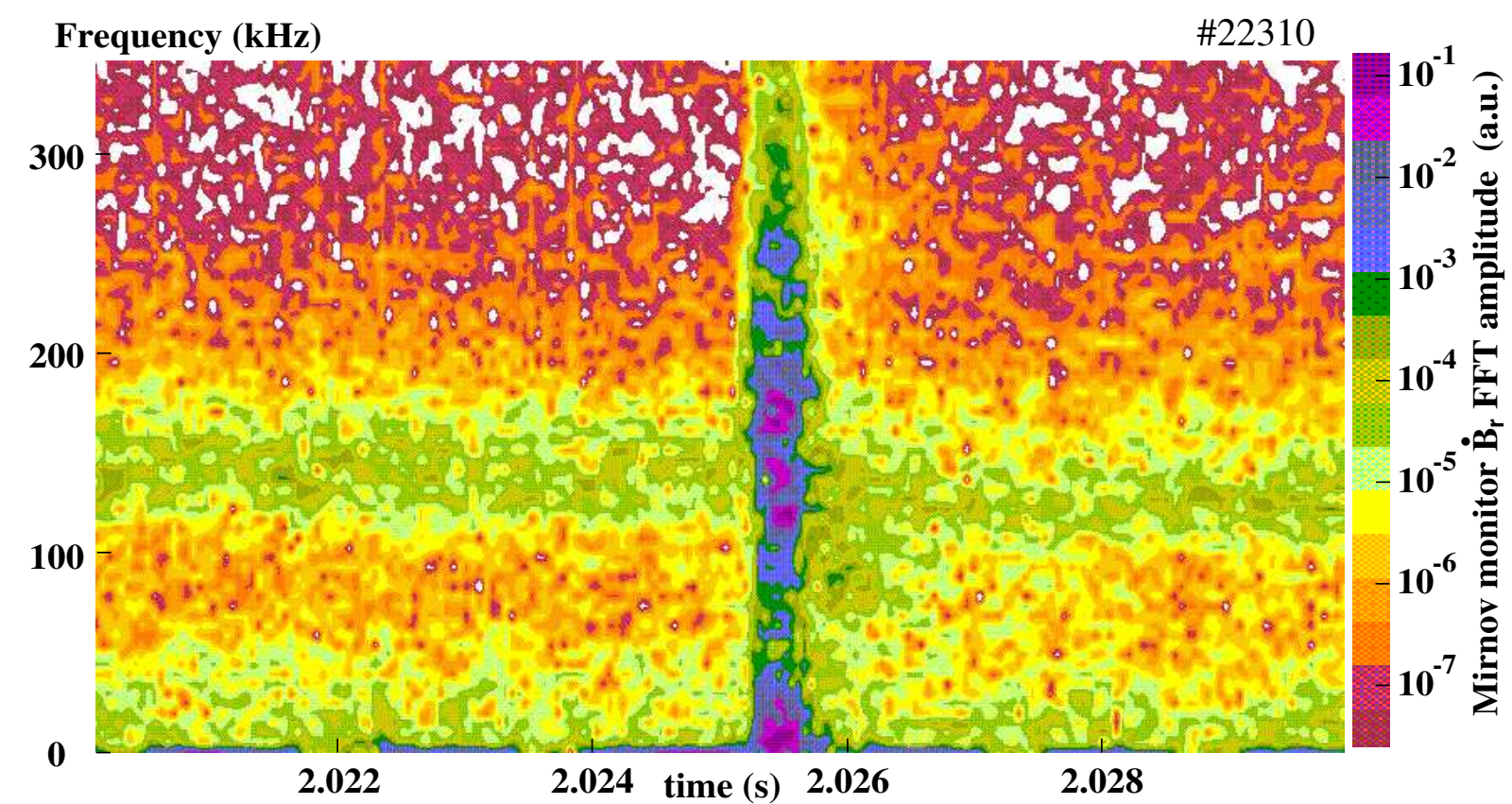

Figure 5: Frequency amplitude evolution derived from a horizontal mid plane LFS Mirnov coil signal. The pellet impact $(880 \mathrm{~m} / \mathrm{s}$ example shown in the right part of figure 2 ) is clearly visible. The broad band intermediate-frequency activity around $150 \mathrm{kHz}$ is likely drift Alfvén turbulence driven TAE at the plasma edge in the vicinity of $\rho_{\text {pol }}=0.8$. Present before and after, the pellet just enhances its amplitude transiently by a factor exceeding 100 .

further, more detailed analysis is under way to identify the connection between this TAE mode close to the edge, excited strongly by the pellet, with the actual seed perturbation in ELM-prone regimes.

Of course data derived from this pellet parameter scan do not allow to differentiate between the influence of different plasma parameters. However, the increase of $\left\langle\dot{B}_{r p}\right\rangle$ with penetration depth seems to indicate that higher local plasma energy densities provide the potential for the excitation of larger MHD perturbations, and suggests that also changes in the plasma conditions would modify the MHD response.

An example further substantiating this claim is shown in figure 7, where applied heating power (with a transiently enhanced Ohmic contribution after pellet injection due to the associated cooling and the increased resistivity), ablation and Mirnov monitor signals are plotted for a train of pellets injected during the L-mode power ramp-down phase before discharge termination. Although subsequent, virtually identical pellets penetrate deeper into the plasma, the resulting pellet born MHD perturbations actually decrease with decreasing energy density. In the $\mathrm{OH}$ and L-mode regime, lower local plasma energies result in a reduced MHD excitation directly driven by the pellet perturbation.

The experimental results shown here regarding the directly pellet driven MHD perturbation in edge-stable regimes below the L-H threshold are only a first step towards the detailed characterisation required for an understanding of the effects causing the ELM triggering by a pellet. More refined measurements 


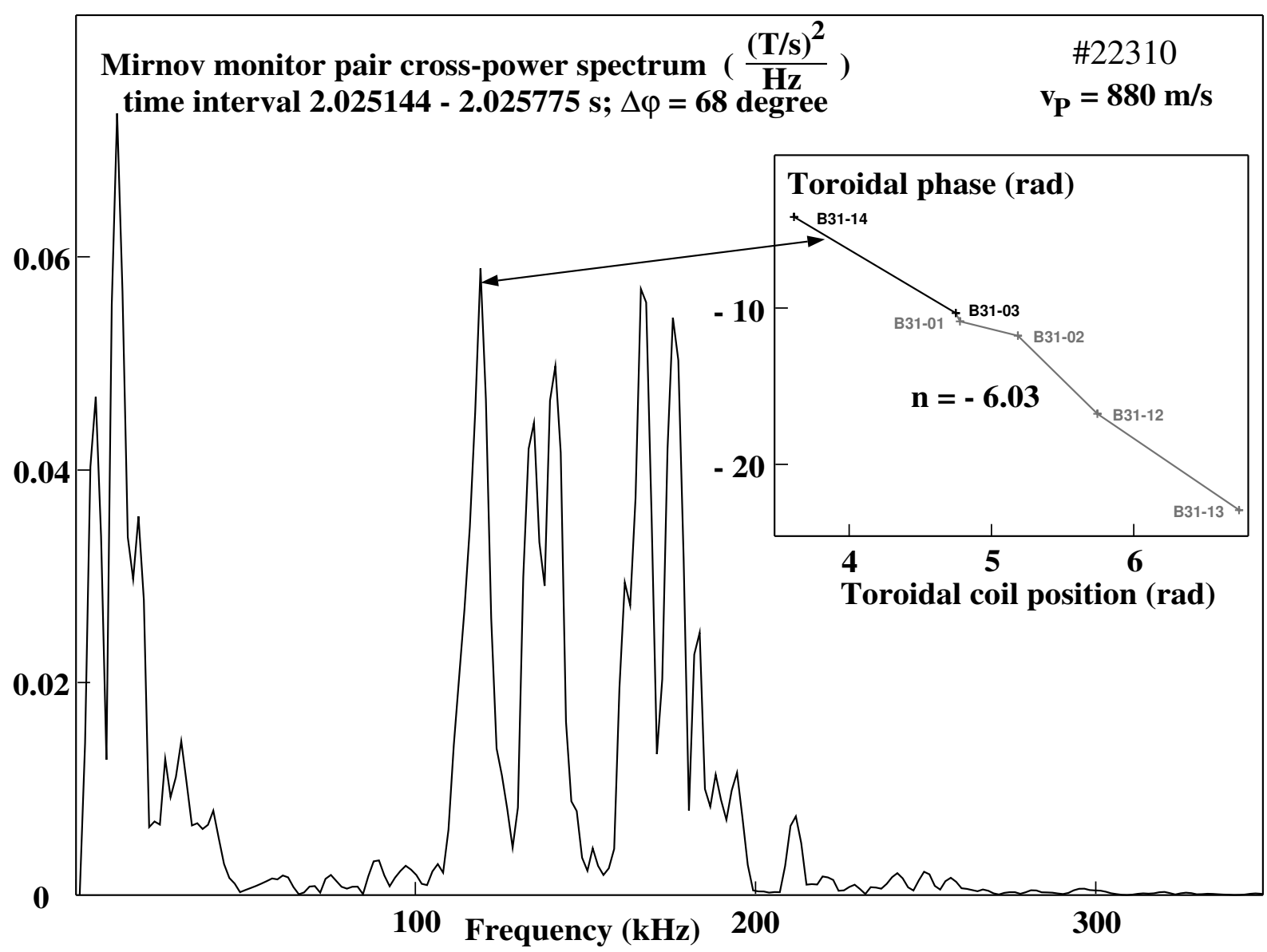

Figure 6: Cross-power spectrum from a Mirnov monitor signal pair [22] located at LFS horizontal mid plane with $68^{\circ}$ toroidal distance. The obvious correlation in both frequency ranges of strong MHD activity is evidence for a coherent mode. Insert: Evolution of the mode number $(\mathrm{n}=6)$ from the toroidal phase distribution for the frequency component referred to by arrow, Mirnov coil pair locations indicated by the black crosses

to resolve details of the spatial and temporal structure of the pellet born MHD perturbation and its correlation with pellet parameters are in preparation on AUG and JET.

However, very recent results obtained during the commissioning phase of the new JET pellet launching system confirm the basic AUG finding, although the lacking pellet quality (fragments and debris coming with the main pellet) does not yet allow for a direct correlation of Mirnov signal magnitude and pellet position. A low frequency $(1 \mathrm{~Hz})$ train of pellets was injected while gradually dropping the auxiliary heating power initiating a $\mathrm{H} \rightarrow \mathrm{L}$ transition. In the L-mode phase, still strong pellet driven MHD activity was detected reaching a magnitude exceeding that one observed at the onset of spontaneous and triggered ELMs during the preceding H-mode phase.

4.2. Pellet perturbation as probe for the edge stability: regimes above the $\mathrm{L} \rightarrow \mathrm{H}$ threshold As previously described, we re-investigated recent experiments performed on AUG and JET with respect 


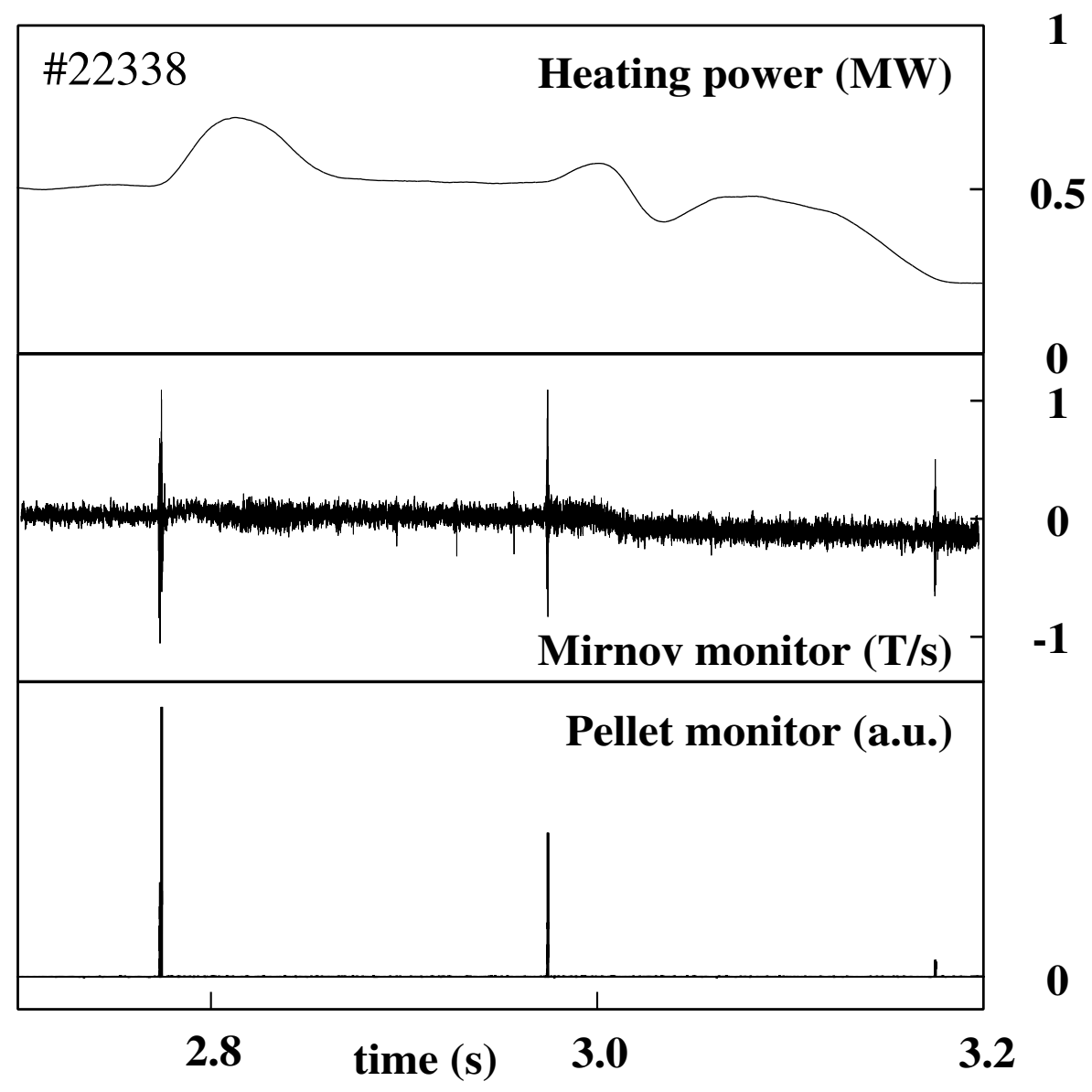

Figure 7: Train of three identical pellets (small $240 \mathrm{~m} / \mathrm{s}$ ) injected during the L-mode power ramp down phase before discharge termination. Although subsequent pellets penetrate deeper, the resulting MHD perturbation decreases with heating power. The transient impact of the pellets on plasma parameters causes enhanced Ohmic heating.

to the pellet's potential for mode destabilisation in the regimes beyond the L-H threshold. Following the typology described in [9] we discuss first the type-III regime.

\subsubsection{Cold edge, resistive type-III regime}

For separatrix heat fluxes just above the L-H threshold tokamak plasmas usually are in the type-III ELM regime. For operational reasons discharges are often driven at first into the low density type-I ELM regime by a step increase of the heating power, then eased more gently into the type-III ELMy regime by strong gas puffing. The evolution of such a discharge run in AUG is shown in the left part of figure 8. Constant strong gas puffing is applied to the steady state type-I ELMy H-mode causing edge cooling and hence an increase of resistivity. This finally results in a transition to the type-III ELM regime; the type-III character is confirmed by the frequency power spectrum of the MHD ELM precursor and the inverted relation between heating power and $f_{E L M}[9]$. Throughout this transition we performed low frequency injection of rather large pellets. Details of the ELM response to pellet injection for both ELM types are 
shown on an expanded time scale in the right part of figure 8. During the type-I phase (discussed in detail later), both spontaneous and triggered ELMs show a fast onset, as can be seen from the burst-like MHD signature and the fast-rising $D_{\alpha}$ radiation from the outer strike point region. It should be noted that the triggered ELM appears to be somewhat enhanced by the large transient particle and energy throughput connected to the pellet ablation - an effect vanishing when the pellet mass is reduced [14]. Spontaneous ELMs in the type-III regime display a significantly slower MHD evolution than in the type-I regime. Triggered ELMs in the type-III regime still show a fast MHD onset (data shown are taken from a twin discharge) while keeping a type-III like divertor heat load pattern. For the type-III ELM case shown here the pellet arrives in a truly quiescent phase between subsequent type-III ELMs. Such a clear separation between triggered and spontaneous ELMs - generally the case in type-I regimes - occurs only rarely in type-III situations, due to their higher natural repetition frequency and the longer duration of the individual events. Nevertheless, results indicate that in the high edge resistivity type-III regime - where spontaneous ELMs are characterized by precursor activity and/or a slow growth - the pellet triggering results in a significantly faster growth either by skipping the precursor stage or causing a faster mode growth.

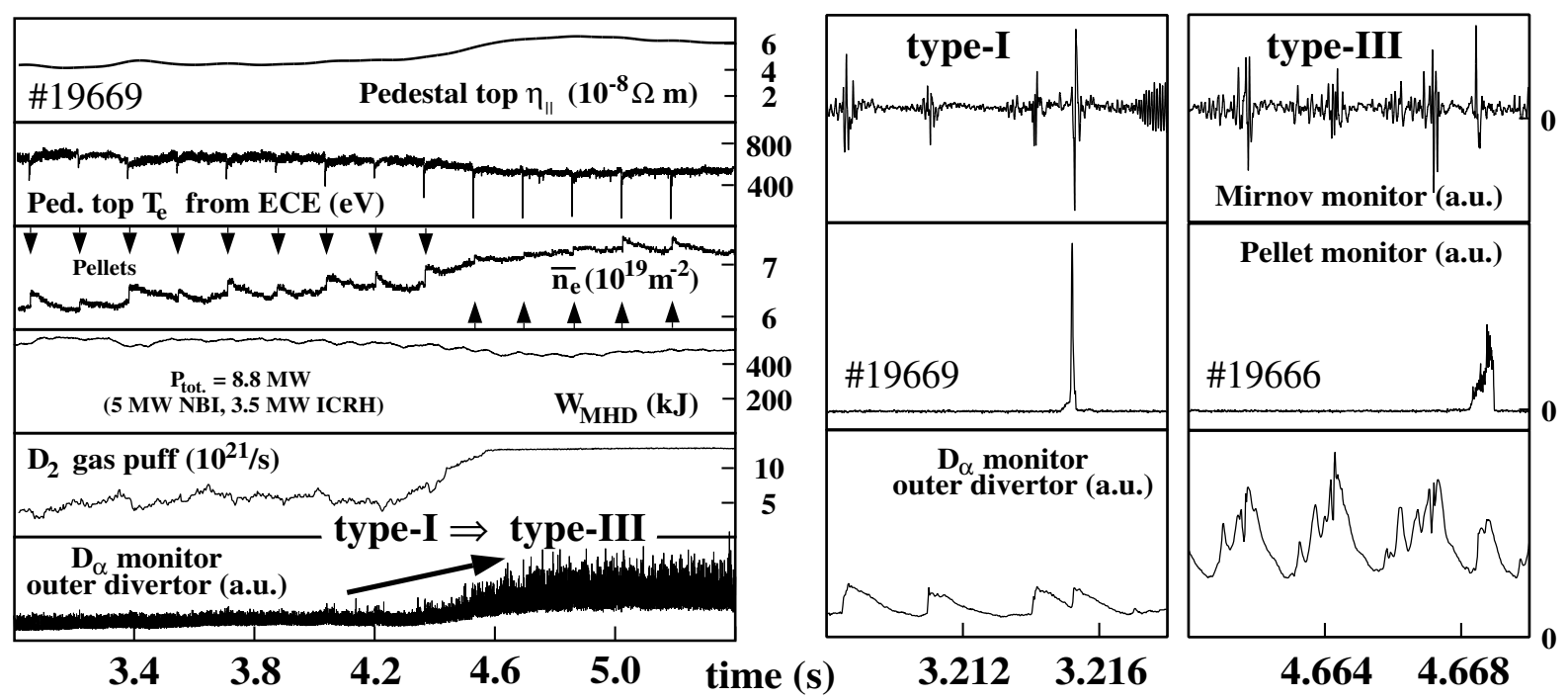

Figure 8: Comparison of spontaneous and triggered ELMs in the type-III regime. A stable type-I H-mode is gradually driven into the type-III regime by the strong gas-puff cooling of the edge and the increase of its resistivity (parallel Spitzer resistivity taking electron data and assuming $Z_{\text {eff }}=1$ )(left). While in the type-III regime spontaneous ELMs typically display a precursor phase and a slow growth, triggered ones evolve much faster by skipping the precursor activity (expanded time scale on far right, data from virtually identical twin shot). They appear more alike to fast growing triggered and spontaneous ELMs in the type-I regime (center).

\subsubsection{The (transient) ELM-free regime}

The type-III and the type-I regimes are separated by the ELM-free regime. Its operational window is usually quite narrow and its appearance transient. ELM-free phases are not stationary since e.g. contin- 
uous profile evolution and/or impurity accumulation finally terminate these phases. As a consequence, for example, integrated radiative high performance scenarios established at AUG for operation with a full tungsten wall coating of plasma facing components required pellet ELM pacing to keep them in the type-I regime with a sufficiently high $f_{E L M}[3]$. Otherwise, a too low or vanishing ELM frequency results in a radiative instability leading to short $\mathrm{H} \rightarrow \mathrm{L}$ back transitions.

Longer lasting but still not steady-state ELM-free phases where observed during investigations performed at JET. Pellet fuelling attempts into hot-ion JET discharges with naturally emerging distinct ELM-free phases showed that every pellet launched caused the appearance of an ELM [23]. A recent detailed re-analysis of JET pellet experiments performed with the explicit aim of ELM triggering [7] confirmed and broadened this finding. An ELM can be triggered any time in an ELMing or ELM-free H-mode phase. Often, the entire ELM behaviour is altered and a train of ELM follows after pellet injection. This is attributed to the pellets' fuelling impact causing a significant change of plasma parameters and transport characteristics at the edge as a similar behaviour found in AUG [14] could be accounted for by this effect. Again in confirmation of AUG findings, also at JET the initial pellet trigger is found not to be caused by the global fuelling effect but by the prompt local perturbation imposed. The triggered ELM becomes already visible when only a tiny mass fraction (about $1 \%$ ) is ablated and the pellet is still in the edge barrier region and has not yet changed the global plasma parameters significantly. Thus, all recent investigations showed that the response to pellet injection does not noticeably differ between ELM-free and type-I ELM phases. Hence, with respect to ELM triggering, the ELM-free regime can be considered as type-I phase with a vanishing natural ELM rate.

\subsubsection{The standard hot edge type-I ELM regime}

The current ITER baseline scenario is the H-mode regime with a hot edge and clear type-I ELMs. In this operational mode the uncontrolled ELMs are expected to cause strong energy bursts whose impact would reduce the divertor lifetime in an unacceptable way [1]. Driven by this concern, most investigations on ELM pacing and pellet triggering of ELMs have been performed in this regime. Following first pellet ELM pacing and mitigation proof-of-principle experiments also investigations dedicated to the physics of the trigger process were made. To provide stable and robust target plasmas with well separated spontaneous ELM events, such investigations were performed at AUG in the perturbative limit, where the pellet rate was small compared to the spontaneous ELM repetition frequency and also small enough to avoid any lasting pellet impact on the global plasma parameters. Every pellet therefore probes the response of the unperturbed target plasma in an arbitrary phase of its natural ELM cycle. On account of the practical importance, pellet triggering investigations in the type-I ELM regime are so far the most advanced ones, so that this study can be regarded as an extension of these previous investigations. We therefore recapitulate here just their main results, described in more detail in [15] to put them into the context of a broader view on the pellet triggering of ELMs or MHD activity in general.

Spontaneous ELMs in the hot edge type-I regime show (mentioned before, see figure 8) a rise time as fast as triggered ELMs. For an ensemble of spontaneous ELMs we derived a value of $67 \pm 22 \mu s$ for this growth time, for an ensemble of triggered ELMs under identical plasma conditions a virtually identical value of $72 \pm 22 \mu \mathrm{s}$. Also, no other significant differences have been observed between triggered and spontaneous 
ELMs in the MHD dynamics at onset yet. However, the transient nature of the event makes their analysis difficult. More detailed investigations with better adapted analysis tools are under way, e.g. of the onset characteristics and the toroidal and poloidal mode numbers of the corresponding MHD perturbations by wavelet analysis [24], with results to be published elsewhere.

For small pellets with sufficiently little impact on the discharge fuelling (typically the pellet mass shall not be larger than the ELM induced particle loss of a spontaneous ELM in this regime) we find also no differences during the later stages in the global appearance, dynamics, duration and spatial distribution of the divertor power flux, if we compare spontaneous and triggered ELMs with the same repetition frequency. Also, the mode and turbulence spectra are quite similar. Essentially, apart from the clearly enforced onset, triggered and spontaneous ELMs appear virtually indistinguishable [25, 26].

Specific AUG investigations [15] of the nature and dynamics of the trigger process showed that the pellet-imposed local perturbation triggering the ELM onset is imposed within the edge transport barrier but clearly inside the separatrix. A delay is observed between the implementation of the initial local perturbation and the ELM onset of about $50 \mu s$ - a value coinciding, as already mentioned, with the observed decay time of a purely pellet driven MHD perturbation in $\mathrm{OH}$ phases. No significant spatial correlation was found between the pellet injection position and the poloidal or toroidal location at which a first ELM indication was found on a Mirnov coil. The MHD signature of every ELM appears over the entire edge region within about $20 \mu s$ (for reference: fast shear Alfvén wave transit time $\frac{2 \pi R_{0}}{v_{A}} \approx 1 \mu s$ ).

\subsubsection{The type-I ELM regime with a radiatively cooled resistive edge}

Experiments on AUG controlled edge and hence divertor cooling by Ar seeding were reported in detail in [3] in which transient ELM-free phases were avoided by applying pellet pacing. Whereas usually the pellet pacing frequency sets the ELM frequency, a higher ELM frequency established itself in several of these discharges $\left(f_{P}\right.$ about $40 \mathrm{~Hz}, f_{E L M}$ about $\left.80 \mathrm{~Hz}\right)$. In such discharge phases both spontaneous and triggered ELMs occurred. As reported in [3], again no significant difference was found between the global action of both ELM types. During the Ar seeding phase a significant reduction of the deposited power density along strike point contours was found. Following Kallenbach's analysis [3], the power load close to the outer strike point was investigated separately for the two kinds of ELMs.. The refined analysis is shown in the left part of figure 9. Triggered and spontaneous ELMs showed an identical behaviour within their individual scatter (boxes representing the $2 \sigma$ width of an ensemble of ELMs recorded during one stable steady state pacing phase) but a significant reduction in power flux with respect to the reference (non-seeded) type-I ELMs. A striking difference is found, however, in the MHD onset dynamics. This is illustrated by the right part of figure 9, showing for each type both signal traces of typical single events and the values obtained by averaging over the entire ensemble. Whereas the spontaneous ELMs grow significantly slower in the cool edge regime $(154 \pm 68 \mu \mathrm{s})$, triggered ones $(60 \pm 38 \mu s)$ keep the fast MHD onset observed in the hot edge case. This confirms the observations made in the comparison of triggered and spontaneous ELMs in type-III and hot edge type-I plasmas. In the low resistivity hot edge mode case spontaneous and triggered ELMs evolve equally fast, obviously, as the growth rate of a spontaneous ELM is so large that even the finite-size seed pellet perturbation cannot accelerate the ELM dynamics 
any further. Increasing edge resistivity results in a reduction of the spontaneous ELM growth rate. In this case, forcing of the ELM by a pellet restores the fastest possible growth leading to a different ELM dynamics.

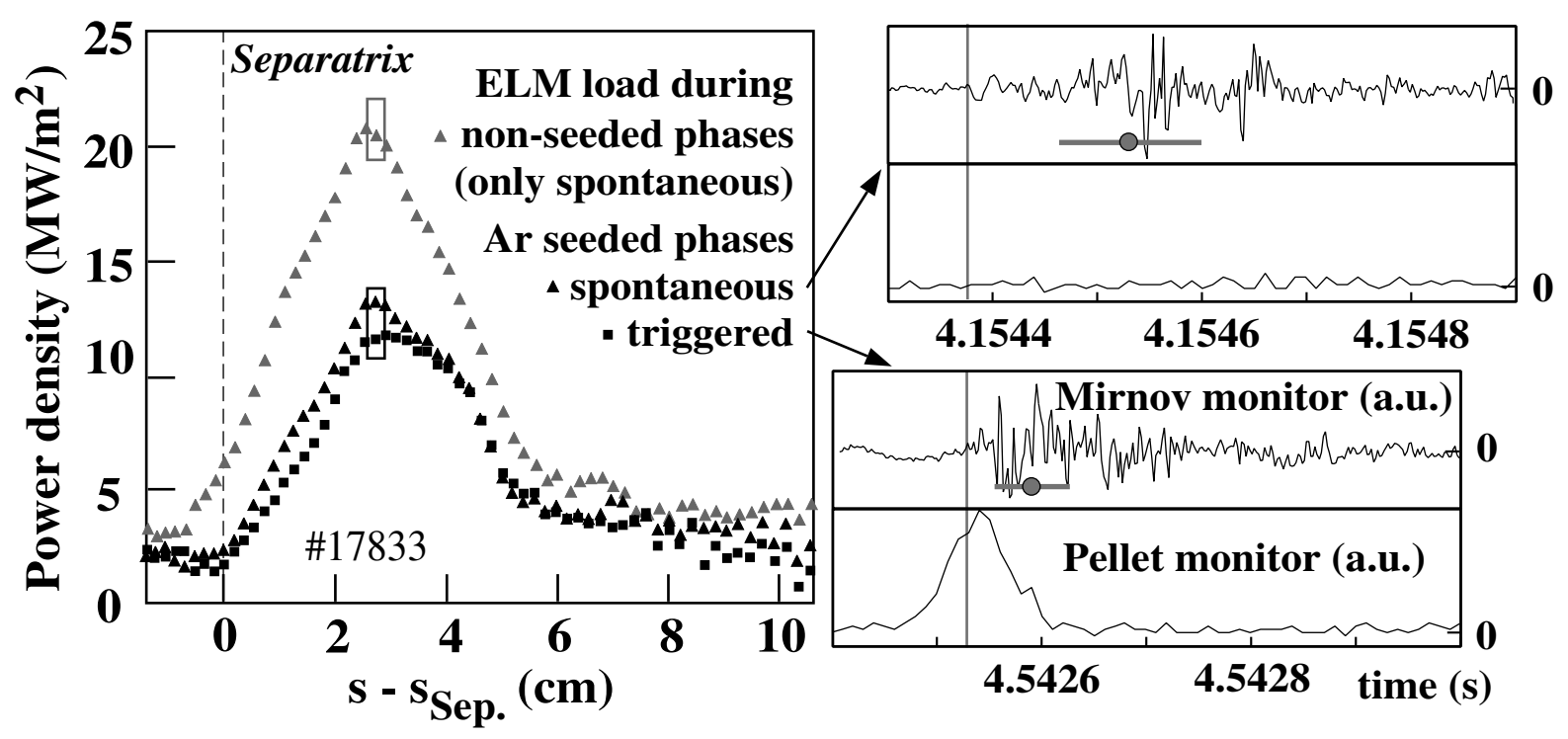

Figure 9: Comparison of spontaneous and triggered type-I ELMs in radiatively cooled AUG edge phases. Whereas there is no difference in their global action like e.g. the imposed heat load (left) the onset dynamics as characterised by the mode amplitude (right) is different. Pellet triggered events show significantly faster growth to maximum amplitude $(60 \pm 36 \mu \mathrm{s}$ for the entire sample as indicated) than their spontaneous counterparts $(154 \pm 68 \mu \mathrm{s})$. This restores the fast ELM growth typical for the hot edge type-I regime. The Ar seeding results in a significant heat flux reduction on the divertor (left, boxes representing standard deviation of entire sample of events).

\subsubsection{The quiescent H-mode}

The above view on the type-I ELMy H-mode regime concludes the examination of the baseline regimes appearing in a scan of separatrix power flux. It was found that every pellet injected could trigger an ELM, which always showed the fastest evolution observed under any conditions in plasma discharges of the respective type. Obviously additional information on the pellet perturbation/ELM trigger mechanism from other high confinement regimes, accessible only by changing key discharge parameters (shaping, pumping, divertor configuration, heating configuration, ramp-up scenario ...) would be extremely helpful to get insight in the physics of the trigger process. Unfortunately little information is available as yet for such regimes, with the exception of the QH-mode in AUG. The QH-mode is a quite interesting regime with respect to edge instabilities and related energy bursts. It displays no strong burst-like transport but the presence of an edge harmonic oscillation (EHO). While the QH regime's very narrow operational range is a clear disadvantage with respect to operational considerations it is nevertheless of great interest to investigate the pellet impact. Results shown here are taken from an experiment aiming to fuel QH plasmas using pellets; details can be found in [27]. For this reason, the pellet size chosen was rather 
large. The temporal evolution of a discharge showing a transition from type-I to $\mathrm{QH}$ without significant changes of global plasma parameters is plotted in the left part of figure 10. A Mirnov monitor signal blow-up at the top shows the EHO with a fundamental frequency of about $10 \mathrm{kHz}$, characteristic for the QH phase. The right part of figure 10, shows a comparison of a spontaneous type-I ELM with the consequences of a pellet impact on a $\mathrm{QH}$ discharge. The pellet drives a direct magnetic perturbation like in the sub-H-mode regimes, but - despite its large size - does not trigger an ELM. Consequently, there is also no detectable global energy release as would be expected in case of the triggering of an ELM. The slight erosion of the pedestal is attributed to the observed transport enhancement following from the massive profile perturbations caused by the pellet injection - an effect well known from pellet fuelling experiments [28]. According to this experiment a pellet cannot trigger an ELM in the QH-mode, a regime obviously not providing conditions where the pellet imposed perturbation can seed an instability subsequently growing into a macroscopic MHD perturbation.

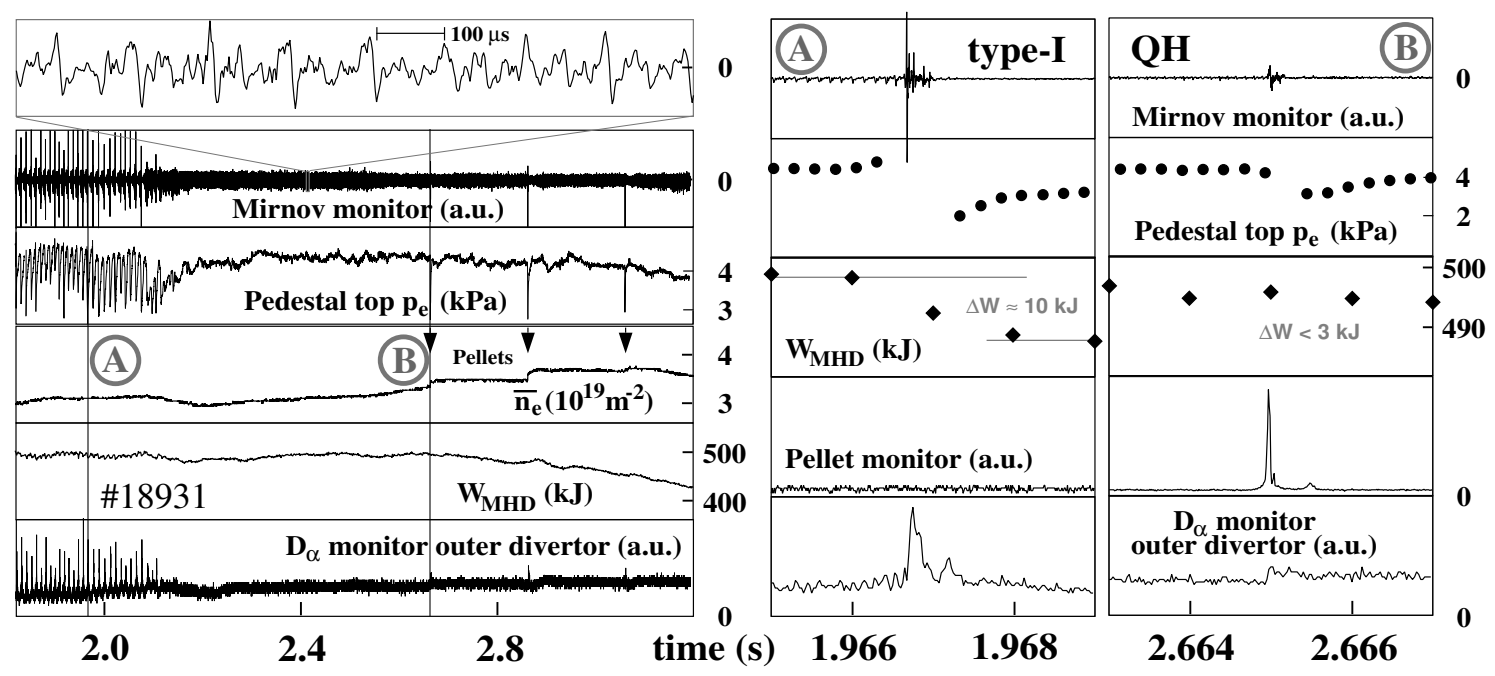

Figure 10: Pellet injection into QH-mode phases does not result in the triggering of an ELM like event. Left: a discharge in which the transition from the type-I ELM regime into a $\mathrm{QH}$ phase is achieved while keeping plasma parameters virtually identical. In the course of this transition, burst-like ELM events are replaced by EHOs at about $10 \mathrm{kHz}$, typical for the QH-mode (blow-up on top). A pellet injected during the QH phase (right, indicated by $\mathbf{B}$ in the left part) does not trigger MHD activity similar to a type-I ELM (middle, A). Furthermore, no significant energy loss is found as expected in the case of triggering.

\section{Discussion}

The investigations made during this study shed new light on the present model of the origin and the dynamics of ELMs. The occurrence of ELMs is usually attributed to the peeling-ballooning stability boundary touched or trespassed over a region slightly inside the separatrix. Once the mode has become potentially unstable, fluctuations of the local plasma parameters in the region with the highest mode growth rate form the microscopic seed perturbations which evolve into the macroscopic ELM instability that ultimately results in a fast transient collapse of the edge transport barrier. The corresponding losses 
of pressure, particles etc. are believed to cause the relaxation of edge parameters back into a stable regime, till radial transport replenishes the edge region to initiate the next ELM cycle.

The observation that a strong local perturbation can trigger an ELM at virtually any instant during this cycle is difficult to reconcile with this conventional picture. It would imply that during the in-between ELM phases the spatio-temporal evolution of the plasma profiles (essentially pressure and current density) were to track closely the marginal stability condition, without any visible manifestation of macroscopic perturbations enforcing this.

In the light of the evidence provided here we consider it more probable that the plasma spends the largest part of the ELM-cycle in a linearly stable, but nonlinearly unstable state, where the finite helical perturbation provided by the pellet cloud constitutes the non-linear trigger. In this context it should be recalled, that also NTMs - the classical tokamak-example of a nonlinear instability - can be reliably triggered by pellet injection [29]. Also the formation of snakes by deeply penetrating pellets is most probably the manifestation of the pellet-induced transition to a second, at least meta-stable helical equilibrium state, caused by the perturbation of pressure or current distribution. In contrast to the case of the ELM, however, the helical structure in the latter two cases must correspond to ideal MHD stable equilibrium states, forming and developing on a time scale (for ASDEX Upgrade conditions) of several tens of ms.

In such a non-linear model, the growth rate of the ELM would then depend, besides on the plasma profiles, also on the instantaneous amplitude of the perturbations, as in fact postulated by the model of Wilson et al. [30]. The spontaneous growth of intrinsic ELMs would be understood as the ultimate arrival of the plasma profiles in a state where the size of the required trigger-perturbation tends practically to zero, so that any statistical fluctuation initiates the breakdown. This picture would, in particular, explain the observation in type-III regimes, where spontaneously arising ELMs grow with a much slower initial rate than those triggered by the finite-size pellet perturbation.

Evidently it is of significant practical importance to understand the creation of the seed perturbation by the pellet and to quantify the requirements on its size for initiating an ELM, if we envisage triggering respectively pacing ELMs at a larger size tokamak, and in particular ITER. For this we must know both the physics of pellet ablation and of the ELM trigger. This was, in particular the motivation to study also the MHD behaviour following pellet injection into ELM-resilient regimes.

When a deuterium pellet enters the plasma it is exposed to hot energy carriers which erode its surface strongly as the sublimation energy of the hydrogen isotope ice is very low $\left(\epsilon_{s u b} \sim 0.005 \mathrm{eV} / \mathrm{particle}\right)$. Accordingly a spherically expanding neutral cloud forms around the pellet [31], which gradually becomes ionized. In this latter stage the cross field expansion of the cloud is stopped and the plasmoid is stretching out along the field lines $[32,33]$. The bright, luminescent part of the plasmoid characterized by its $D_{\alpha}$ radiation has an extension along field in the order of $10 \mathrm{~cm}$ [34]. At its edge, the radiation signal disappears due to the vanishing of the neutral density [6]. Even beyond this visually striking structure observed during the ablation the field aligned cloud expansion continues [35, 36]. 
Due to the differences between the characteristic propagation speeds of the electron heat pulse, the magnetic perturbations and the ion sound wave, the perturbations will develop on different time scales. Along the field lines and ultimately within the whole flux surface, the electron energy will concentrate in the small region filled by the newly ablated material, causing a strong pressure enhancement there, but a general pressure reduction over the rest of the surface. This electron temperature decrease can be clearly observed within a few $10 \mu \mathrm{s}$. It should be noted that this process per se does not lead to a change in the total energy content of the flux surface and hence in the flux-surface averaged pressure, although the concomitant atomic loss processes (ionization, CX, radiation) will do so. The resulting inhomogeneous pressure along field lines will ultimately equilibrate via plasma flow on an ion-sound time scale, but force balance will require a much faster reaction exciting a broad band of Alfvén type waves, damped by dissipation and continuum damping. This Alfvénic activity will be particularly visible in frequency and n-number regions with little damping, which explains the similarity in spectrum (albeit a factor 100 increase in amplitude) to the pre-pellet activity, illustrated, e.g. by Fig. 5. This activity is therefore an indicator of the magnitude of the equilibrium perturbation caused by the pellet, but a manifestation rather than the cause of the processes leading to the ELM.

Standard theories postulate ELMs to be due to pressure or current profile driven instabilities, and we want to examine the possibility that the pellet initiates such a helical mode with a finite amplitude. As mentioned above, the pellet will clearly cause a poloidally and toroidally asymmetric plasma pressure perturbation, which will persist over some ion transit time, i.e. a $100 \mu s$ scale, commensurate with an ELM growth rate. It will also cause a displacement of the parallel currents out of the flux tube passing through the pellet cloud, which could be viewed as a helical current perturbation. However, the skin effect will attempt to screen this current perturbation from the surrounding, so that it should become visible and active only on a resistive time scale estimated to be in the several ms range. Clearly the pressure perturbation, as the helical, finite amplitude seed of a pressure driven mode appears a strong candidate for the explanation of the pellet effect.

An alternative explanation to be considered is the change in stability properties due to the modification of the axisymmetric profiles of the plasma parameters [37, 38], driving the latter over the brink of the linear stability threshold. In the straightforward form this model would appear to have difficulties to explain the apparent independence of the trigger process on ELM phase and pellet perturbation magnitude, particularly in view of the fact that a pellet changes less the average pressure on a surface than its distribution. Nevertheless, it has to be noted that the evidence for ELM triggering by small pellets stems exclusively from high-field side pellet injection experiments, which produce, on the low field side a fast and significant pressure reduction over the whole flux surface. On the low field side, the pellet ablation looks therefore like a rare fraction wave propagating inward with approximately the pellet penetration speed (a few cm per $100 \mu s$ ), which transiently causes locally very strong pressure gradients. A theory of pressure driven modes for non-equilibrium situations with non-constant pressures on a flux surface is evidently not available, but one would expect the low-field side gradients to be relevant for ballooning instability. Evidently, forthcoming experiments with different injection geometries will be very informative in this 
respect.

Of particular interest in this context are the observations in the QH-mode [27], which in the frame of our model should correspond to a particular class of plasma profiles (requiring for their attainment also special external measures) where the development into a nonlinearly strongly unstable state is prevented, possibly by the self-regulating action of the observed EHOs. Evidently this hypothesis raises the interest into future experiments with pellet injection into other regimes, like the situation where ELMs are suppressed by the action of resonant magnetic perturbations. The absence of an immediate ELM-trigger by an injected individual pellet in such a case would be taken as a confirmation that the externally imposed field perturbations keep plasma profiles away from non-linearly unstable situations. A delayed reaction would have to be interpreted as the result of the pellet-induced changes in the axisymmetric profiles [39].

A host of future experiments and measurements could support or disprove the above model, by offering more detailed insight in the underlying dynamics. Such kind of information might become available from a better resolution of the temporal and spatial origin of the triggered ELM. Eventually detailed investigations of the observed delay time between ELM onset and imposed pellet perturbations at different poloidal position might help to identify the detailed trigger mechanism. Both the perturbation effect of the pellet and the edge stability characteristics can be sounded out by launching pellets into plasmas in different regimes, but also during phases of active manipulation of the plasma edge e.g. by enhanced ripple [40], edge ergodisation [41, 42] or fast plasma motions [43, 44]. And finally, the impact of pellet pacing on edge profiles and its self-consistent feedback achieving steady state conditions can provide valuable information on the trigger process and its limitations. There are e.g. indications from AUG pacing investigations that while the driven $f_{E L M}$ can be imposed instantly, a finite response time is required (in the order of about $100 \mathrm{~ms}$ ) to achieve actual ELM mitigation and to re-establish the relation $f_{E L M} \times \Delta_{E L M}=$ const. [2]. It will also be of interest if and how the ELM triggering behaves when applied over a broader operational space, especially to situations with a larger - and hence more ITER relevant - frequency enhancement ratio between the driven and spontaneous ELMs.

The questions that have ultimately to be answered are: is successful ELM pacing by pellets possible at ITER and if yes what are the optimized pellets parameters, particularly in view of the fact that the pellet pacing system will make a - possibly unwanted - contribution to refuelling. At present there appears to be quite some headroom available to reduce the associated fuelling contribution, as the imposed MHD perturbations seem to depend on penetration depth but not on the ablation rate. ITER could therefore go for faster pellets to reach the required penetration depth with pellets of sufficiently small mass. Besides reducing energy losses associated with the imposed enhanced convection this could also ease the pumping load, currently a main concern at ITER.

Upcoming investigations at AUG with its new re-located blower gun [45], at JET - now equipped with a novel specially tailored injection system [8]- and at DIII-D with the new pellet dropper installed [46] are expected to clarify many of these questions. 


\section{References}

[1] FEDERICI, G. et al., Plasma Phys. Controlled Fusion 45 (2003) 1523.

[2] Herrmann, A., Plasma Phys. Controlled Fusion 44 (2002) 883.

[3] KALLENBACH, A. et al., J. Nucl. Mater. 337-339 (2005) 732.

[4] ASDEX Upgrade Team, Fusion Science and Technology 44 (2003) 569.

[5] ANDELFINGER, C. et al., Rev. Sci. Instrum. 63 (1993) 983.

[6] KOCSIS, G. et al., Rev. Sci. Instrum. 75 (2004) 4754.

[7] LANG, P. T. et al., Nucl. Fusion 47 (2007) 754.

[8] GERAUD, A., The JET high frequency pellet injector project, in 24th SOFT Conference, Warsaw, 2006, pages $\mathrm{P} 4-\mathrm{H}-201,2006$.

[9] ZOHM, H., Plasma Phys. Controlled Fusion 38 (1996) 105.

[10] CONNOR, J. W. et al., Phys. Plasmas 5 (1998) 2687.

[11] WILSON, H. et al., Nucl. Fusion 40 (2000) 713.

[12] SNYDER, P. B. et al., Plasma Phys. Controlled Fusion 46 (2004) A131.

[13] MC NEILL, D., J. Nucl. Mater. 162-164 (1989) 476.

[14] LANG, P. T. et al., Nucl. Fusion 43 (2003) 1110.

[15] KOCSIS, G. et al., Nucl. Fusion 47 (2007) 1166.

[16] BELONOHY, E. et al., Nucl. Fusion 48 (2008) 065009.

[17] LOREnZ, A. et al., Fusion Eng. Design 69 (2003) 15.

[18] MARASCHEK, M. et al., Phys. Rev. Lett. 79 (1997) 4186.

[19] SCOTT, B., Plasma Phys. Controlled Fusion 39 (1997) 471.

[20] CAmargo, S. J. et al., Phys. Plasmas 3 (1996) 3912.

[21] BOlzonella, T. et al., Plasma Phys. Controlled Fusion 46 (2004) A143.

[22] STRAIT, E. J. et al., Plasma Phys. Controlled Fusion 36 (1994) 1211.

[23] ALPER, B. et al., ELMs, precursors and triggers on JET, in Europhysics Conference Abstracts (CD-ROM, Proc. of the 29th EPS Conference on Plasma Physics and Controlled Fusion, Montreux, 2002), edited by BEHN, R. and VARANDAS, C., volume 26B, page P1.025, Geneva, 2002, EPS.

[24] KASS, T. et al., Nucl. Fusion 38 (1998) 111.

[25] LANG, P. T. et al., Nucl. Fusion 44 (2004) 665.

[26] NEUHAUSER, J. et al., Nucl. Fusion 48 (2008) 045005.

[27] SUTTROP, W. et al., Nucl. Fusion 45 (2005) 721.

[28] LANG, P. T. et al., Nucl. Fusion 36 (1996) 1531, Corrigendum in Nucl. Fus. 36 (1996) 153.

[29] ZOHM, H. et al., Nucl. Fusion 41 (2001) 197.

[30] WILSON, H. and COWLEY, S., Phys. Rev. Lett. 92 (2004) 175006.

[31] PARKS, P. B. and TURNBUlL, R. J., Phys. Fluids 21 (1978) 1735. 
[32] DURST, R. et al., Nucl. Fusion 30 (1990) 3.

[33] WURDEN, G. A. et al., Rev. Sci. Instrum. 61 (1990) 3604.

[34] MÜLLER, H. W. et al., Nucl. Fusion 42 (2002) 301.

[35] KAUFMANN, M. et al., Nucl. Fusion 26 (1986) 171.

[36] LENGYEL, L. L. et al., Nucl. Fusion 39 (1999) 791.

[37] MILORA, S. L. et al., Nucl. Fusion 35 (1995) 657.

[38] PEgOuRIE, B., Plasma Phys. Controlled Fusion 49 (2007) R87.

[39] BAYLOR, L. et al., Nucl. Fusion 47 (2007) 1598.

[40] SAIBENE, G. et al., Nucl. Fusion 47 (2007) 969.

[41] MOYER, R. A. et al., Phys. Plasmas 12 (2005) 056119.

[42] LIANG, Y. et al., Phys. Rev. Lett. 98 (2007) 265004.

[43] DEGEling, A. et al., Plasma Phys. Controlled Fusion 45 (2003) 1637.

[44] LANG, P. T. et al., Plasma Phys. Controlled Fusion 46 (2004) L31.

[45] LANG, P. T. et al., Rev. Sci. Instrum. 78 (2007) 023504.

[46] BAYLOR, L. et al., Bull. Am. Phys. Soc. 51, 115 (2006) . 